\title{
High-Pressure Phases of Silane
}

\author{
Chris J. Pickard and R. J. Needs \\ Theory of Condensed Matter Group, Cavendish Laboratory, J. J. Thomson Avenue, Cambridge CB3 OHE, United Kingdom
} (Received 12 April 2006; published 27 July 2006)

\begin{abstract}
High-pressure phases of silane $\mathrm{SiH}_{4}$ are predicted using first-principles electronic structure methods. We search for low-enthalpy structures by relaxing from randomly chosen initial configurations, a strategy which is demonstrated to work well for unit cells containing up to at least ten atoms. We predict that silane will metallize at higher pressures than previously anticipated but might show high-temperature superconductivity at experimentally accessible pressures.
\end{abstract}

DOI: $10.1103 /$ PhysRevLett.97.045504

PACS numbers: 61.66.Fn, 71.20.-b, 74.10.+v

As early as 1935, Wigner and Huntingdon suggested that hydrogen would become metallic under sufficient compression [1]. Despite many attempts to produce metallic hydrogen, it has stubbornly remained insulating in diamond-anvil-cell experiments up to the highest pressure so far achieved of $342 \mathrm{GPa}$ [2]. It has recently been suggested [3] that the group IV hydrides, methane $\left(\mathrm{CH}_{4}\right)$, silane $\left(\mathrm{SiH}_{4}\right)$, and germane $\left(\mathrm{GeH}_{4}\right)$, might become metallic at lower pressures because the hydrogen is "chemically precompressed" by the group IV atoms.

In their recent first-principles density-functional-theory (DFT) study of silane, Feng et al. [4] predicted the stability of a layered structure above $25 \mathrm{GPa}$. This phase was found to be semimetallic at $91 \mathrm{GPa}$, and the density of electronic states at the Fermi energy [5] increased rapidly with further application of pressure. An estimate within the BardeenCooper-Schrieffer (BCS) theory [6] indicated that the superconducting transition temperature would increase from about $0 \mathrm{~K}$ at $91 \mathrm{GPa}$ to about $166 \mathrm{~K}$ at $202 \mathrm{GPa}$, pressures which are comfortably within the range of diamond-anvil cells.

First-principles methods generally give accurate cohesive energies and equilibrium volumes for materials with known crystal structures, but the prediction of stable structures has remained very difficult. It is routine to relax configurations to nearby minima in the potential energy surface, the lowest-energy minimum found corresponding to the most favored structure. However, if none of the initial configurations is sufficiently close to the global minimum, the configurations become trapped in higherenergy local minima. Trapping in local minima is a severe problem, because their number is expected to increase exponentially with the number of degrees of freedom. In the past, this problem has typically been addressed by selecting initial configurations derived from experimental knowledge about the system, the known structures of similar materials, chemical intuition, and results from simpler computational methods. The shortcomings of this approach have led to interest in determining stable structures by combining first-principles methods with more advanced search techniques [7-10].
We have developed a simple strategy for generating essentially random initial configurations. First, we choose the number and identity of the atoms in the unit cell. To form "random" unit cells, we choose three cell-vector lengths randomly and uniformly between 0.5 and 1.5 (in arbitrary units) and three cell angles randomly and uniformly between $40^{\circ}$ and $140^{\circ}$. The resulting cell vectors are scaled to produce the desired volume, which is also selected randomly and uniformly from between 0.5 and 1.5 of some chosen volume. The positions of the atoms are then chosen randomly and uniformly within the cell. Firstprinciples methods are then used to relax each structure to a minimum in the enthalpy. The structures so obtained are liable to be trapped in local minima, and undoubtedly the probability of finding the global minimum will drop rapidly with increasing system complexity. However, for systems of moderate complexity, our approach has a high probability of locating the global minimum. Our approach has been to continue generating configurations until the relaxed structures with low enthalpies are generated several times and, where possible, to look for the occurrence of previously known "marker" structures.

All of the first-principles calculations were performed using a developer's version of the CASTEP code [11]. For the initial search over structures, we used the local density approximation (LDA), for the exchange-correlation functional [12], and default ultrasoft pseudopotentials [13]. The $k$-point sets were generated separately for each unit cell, and no symmetry restrictions were applied. Medium quality Brillouin zone sampling using a grid of spacing $2 \pi \times$ $0.07 \AA^{-1}$ and a plane wave basis set cutoff of $280 \mathrm{eV}$ were found to be sufficient for the initial search over structures. We recalculated the enthalpy curves with higher accuracy using a Brillouin zone sampling of $2 \pi \times 0.03 \AA^{-1}$, harder pseudopotentials requiring a plane wave cutoff of $360 \mathrm{eV}$, and the Perdew-Burke-Ernzerhof generalized gradient approximation (GGA) density functional [14]. Our calculations are for the zero-temperature ground state, and we neglected the zero-point vibrational energy.

We tested our approach on silicon at zero pressure. Using two-atom unit cells and 100 randomly chosen initial 
configurations, we obtained the diamond (15 times), $\beta$-tin (2 times), Imma (14 times), and simple hexagonal (15 times) structures. Both experiment and DFT calculations agree that these are the four lowest-pressure thermodynamically stable phases of silicon [15]. A study of 8-atom unit cells of carbon at $300 \mathrm{GPa}$ generated the diamond structure as the lowest-enthalpy phase in addition to numerous higher-enthalpy phases, including the $\mathrm{BC} 8$ phase, which has been postulated as a stable phase of carbon at TPa pressures [16]. We also studied 16-atom cells of hydrogen at $250 \mathrm{GPa}$, finding, among others, the Cmca molecular phase, which DFT calculations predict to be the most stable at that pressure [17].

Our approach is particularly suitable for predicting stable high-pressure phases because they often have fairly simple structures with a small number of atoms in the primitive cell. In addition, our method does not require the selection of highly system-specific parameter values. Our scheme could be modified to include other features, such as methods for extricating configurations from local energy minima [18]. It is also possible to introduce other constraints on the initial configurations so that one can predict the structures of clusters, defects, surfaces, etc. For example, we have used our approach to search for low-energy self-interstitial defect structures in silicon. To construct the initial configurations, we removed an atom and its four nearest neighbors from a 32-atom supercell and then placed six silicon atoms randomly within the hole. We readily found the split- $\langle 110\rangle$ and hexagonal interstitial configurations, which other DFT calculations have shown to be lowest in energy [19], and found that all other selfinterstitial local minima were at least $0.3 \mathrm{eV}$ higher in energy. The generation of numerous low-energy structures is a useful feature of our strategy, as they give information about the structures which might be formed under other conditions of pressure and temperature or out of thermal equilibrium.

We tested our search strategy for simulation cells containing one, two, and four $\mathrm{SiH}_{4}$ units at $250 \mathrm{GPa}$. With one $\mathrm{SiH}_{4}$ unit per cell, the lowest-enthalpy structure occurred 4 times from 13 initial configurations, but it was $0.36 \mathrm{eV}$ per $\mathrm{SiH}_{4}$ unit higher in enthalpy than the most stable structure found with two $\mathrm{SiH}_{4}$ units per cell, which occurred 8 times from 37 initial configurations. With four $\mathrm{SiH}_{4}$ units per cell, the most stable structure was $0.13 \mathrm{eV}$ per $\mathrm{SiH}_{4}$ unit higher in enthalpy than for the two $\mathrm{SiH}_{4}$ unit cell, and each of the low-enthalpy phases occurred only once from 38 configurations. Clearly, the simulation cells containing one $\mathrm{SiH}_{4}$ unit are too constrained to produce very-low-enthalpy structures, while those containing four $\mathrm{SiH}_{4}$ units have too many degrees of freedom for our search strategy with the number of initial configurations used. We concluded that using two $\mathrm{SiH}_{4}$ units per simulation cell and 40 initial configurations represented an affordable and efficient approach to finding low-enthalpy phases of silane at high pressures.
Further runs were performed at $0,50,100,150$, and $200 \mathrm{GPa}$, with two $\mathrm{SiH}_{4}$ units per simulation cell and 40 initial structures. At $0 \mathrm{GPa}$, the lowest-energy structure could not be determined with confidence because the lowenergy structures were not found repeatedly. This reflects the fact that at zero pressure many different structures have similar energies (within $0.1 \mathrm{eV}$ per $\mathrm{SiH}_{4}$ unit), including those consisting of silane molecules, disilane plus a hydrogen molecule, and various polymeric forms plus hydrogen molecules. The various packings of silane molecules were therefore inadequately explored using 40 initial structures.

Having selected the low-enthalpy phases from the above runs, we recalculated their enthalpies with greater computational accuracy; see Fig. 1. The structures of some of the lower-enthalpy structures found are described in the auxiliary material [20].

An insulating chainlike structure with $P 2 / c$ symmetry was most stable in a small region of pressures around $40 \mathrm{GPa}$, but a structure of $I 4_{1} / a$ symmetry became more stable at about $50 \mathrm{GPa}$. The $I 4_{1} / a$ phase is predicted to be insulating up to about $200 \mathrm{GPa}$, when a conduction band about $3 / 10$ of the way from $\Gamma$ to $Z$ in the Brillouin zone becomes degenerate with the highest-energy valence band state at the $Z$ point. The indirect band overlap increases with pressure but is still only $0.3 \mathrm{eV}$ at $262.5 \mathrm{GPa}$, and the material remains a semimetal with a small density of states at the Fermi energy; see Fig. 2. Given the well-known shortcomings of standard DFT approaches such as the LDA and GGA functionals, which normally underestimate band gaps, it is likely that our calculations underestimate the pressure at which the $I 4_{1} / a$ phase becomes semimetallic and overestimate the density of states at the Fermi energy at higher pressures. The $I 4_{1} / a$ phase remains the lowest-enthalpy phase up to $262.5 \mathrm{GPa}$, at which pressure a structure of $C 2 / c$ symmetry becomes stable. This phase is a good metal; see Fig. 2. Our predicted transition pressure is subject to significant uncertainties from the use of approximate DFT and the neglect of zero-point motion, but it

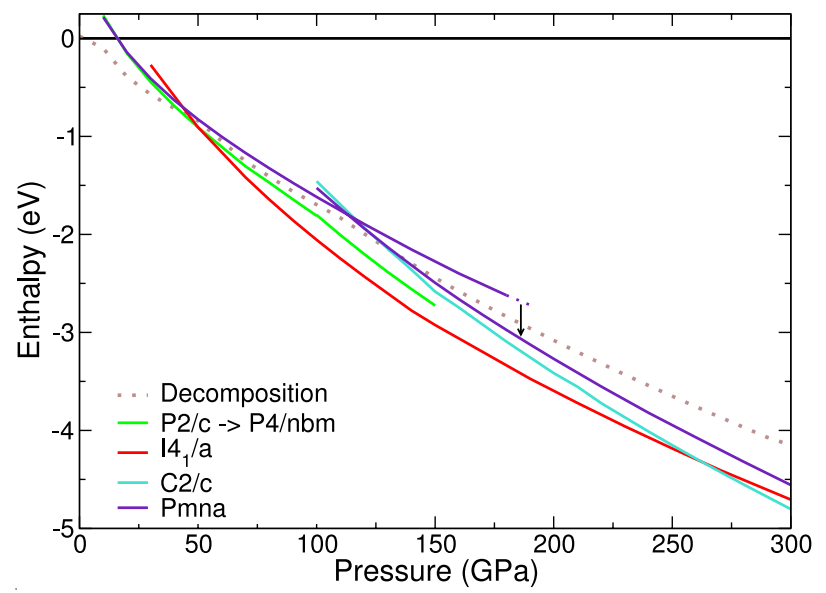

FIG. 1 (color online). The enthalpies per $\mathrm{SiH}_{4}$ unit of various structures as a function of pressure, referenced to the $T 1$ phase of Ref. [4]. 

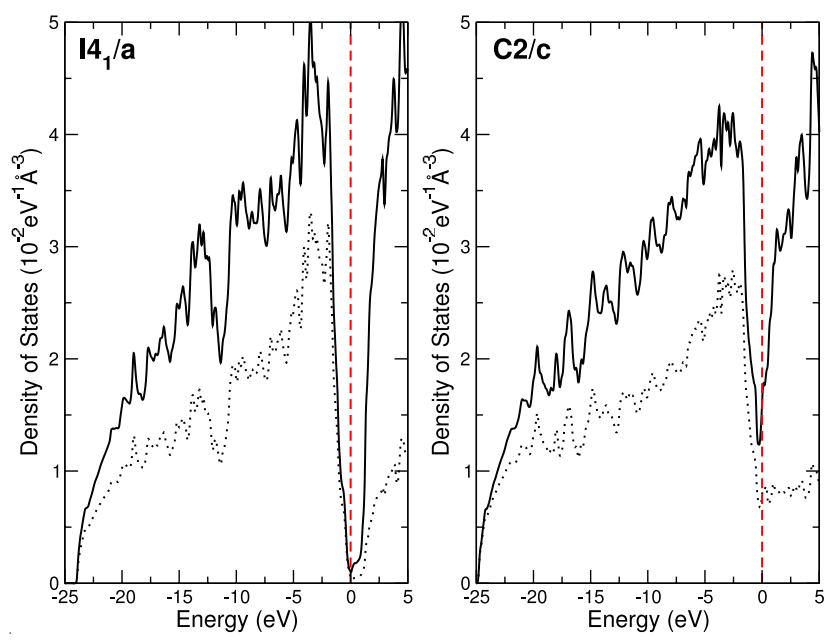

FIG. 2 (color online). Densities of states of the $I 4_{1} / a$ and $C 2 / c$ phases at the coexistence pressure of $262.5 \mathrm{GPa}$. The partial density of states projected onto the $\mathrm{H}$ atoms is shown by dashed curves and the Fermi energies by the vertical dashed lines.

is highly likely that the $C 2 / c$ phase will become more stable than $I 4_{1} / a$ at high pressures because it is denser.

In the $I 4_{1} / a$ structure illustrated in Fig. 3, each Si atom is bonded to $8 \mathrm{H}$ atoms. Each of the $\mathrm{H}$ atoms forms a "bridge" between two neighboring $\mathrm{Si}$ atoms. There are two such bridges between every pair of neighboring $\mathrm{Si}$ atoms, the four atoms forming a $\mathrm{Si}_{2} \mathrm{H}_{2}$ plane. This bonding arrangement consists of two "banana bonds," which are electron-deficient three-center-two-electron bonds, reminiscent of those in $\mathrm{B}_{2} \mathrm{H}_{6}$ (diborane) [21]. The arrangement of the $\mathrm{Si}_{2} \mathrm{H}_{2}$ planes keeps the $\mathrm{H}$ atoms away from each other, and the $I 4_{1} / a$ structure contains only $\mathrm{Si}-\mathrm{H}$ bonds. Each of the $\mathrm{Si}$ sites and the $\mathrm{H}$ sites is equivalent in this high-symmetry structure, which has a 10 -atom primitive

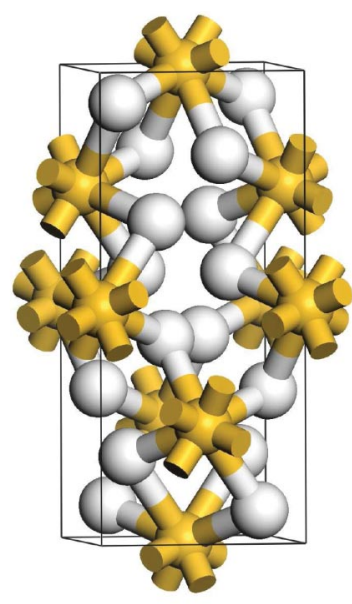

$14{ }_{1} / \mathrm{a}$
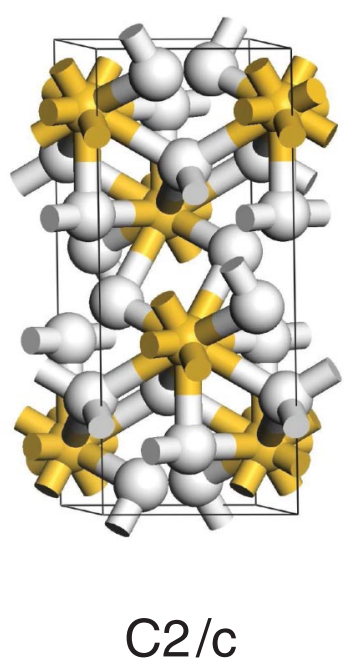

FIG. 3 (color). The $I 4_{1} / a$ and $C 2 / c$ structures. The golden spheres represent silicon atoms, and the white spheres hydrogen. unit cell. The layered $\mathrm{O} 3$ structure proposed in Ref. [4] also contains some bridging $\mathrm{Si}-\mathrm{H}-\mathrm{Si}$ bonds, although it also contains $\mathrm{H}$ atoms bonded to single $\mathrm{Si}$ atoms. Our $\mathrm{I} 4_{1} / a$ structure is about $0.4 \mathrm{eV}$ per $\mathrm{SiH}_{4}$ unit lower in enthalpy than the $\mathrm{O} 3$ structure at $100 \mathrm{GPa}$ and about $0.7 \mathrm{eV}$ per $\mathrm{SiH}_{4}$ unit lower at $180 \mathrm{GPa}$.

In the metallic $C 2 / c$ structure illustrated in Fig. 3, each $\mathrm{Si}$ atom is bonded to $11 \mathrm{H}$ atoms. Each of the Si sites is equivalent, and there are three inequivalent $\mathrm{H}$ sites in which the $\mathrm{H}$ atom forms bonds to two, three, or four $\mathrm{Si}$ atoms. The average coordination number of the $\mathrm{H}$ atoms in the $C 2 / c$ structure is larger than in $I 4_{1} / a$, which accounts for its smaller equilibrium volume. The metallic character of this phase means that the nature of the interatomic bonding is less clear cut than in the $I 4_{1} / a$ structure, but again a description in terms of electron-deficient $\mathrm{Si}-\mathrm{H}$ bonds is appropriate.

Our search revealed a number of other low-enthalpy phases, including the O3 Pmna structure of Feng et al. [4]. We found that, at around $200 \mathrm{GPa}, \mathrm{O} 3$ Pmna converts into a more compressed structure of the same symmetry, but it still has a substantially higher enthalpy than our $I 4_{1} / a$ phase. We followed the more compressed phase to lower pressures and found it to be more stable than the $\mathrm{O} 3$ Pmna structure down to about $110 \mathrm{GPa}$. We also found a low-enthalpy phase with $I \overline{4} 2 d$ symmetry which is only $0.1 \mathrm{eV}$ per $\mathrm{SiH}_{4}$ unit above the most stable phase at $100 \mathrm{GPa}$. The $P 2 / c$ phase mentioned above converts to a structure with $P 4 / \mathrm{nbm}$ symmetry at $100 \mathrm{GPa}$. Interestingly, the $P 4 / \mathrm{nbm}$ phase has a layered structure, like $\mathrm{O} 3$, but with every $\mathrm{H}$ atom participating in a bridging bond, which results in its lower enthalpy.

We studied the stability of the $C 2 / c$ phase to rearrangements of the $\mathrm{H}$ atoms using cells containing four $\mathrm{SiH}_{4}$ units at $250 \mathrm{GPa}$. To make initial configurations, we used the $\mathrm{C} 2 / \mathrm{c}$ Si framework and placed the $16 \mathrm{H}$ atoms randomly within the simulation cell. We relaxed 15 such configurations, finding the $C 2 / c$ structure as the lowest-enthalpy phase, an $F d d d$ structure only $30 \mathrm{meV}$ per $\mathrm{SiH}_{4}$ unit higher in enthalpy, and several other slightly less stable phases. All of the low-enthalpy phases contained a mixture of two-, three-, and fourfold coordinated $\mathrm{H}$ atoms, as in the $C 2 / c$ structure, and they all had very similar volumes and densities of electronic states.

Silane is, in fact, unstable to decomposition into hydrogen and silicon at low pressures, and we therefore considered the thermodynamic stability of our new phases against the formation of hydrogen molecules. At low pressures, we found some low-enthalpy structures containing hydrogen molecules, indicating the instability of silane at low pressures, but none of the low-enthalpy high-pressure structures contain any $\mathrm{H}-\mathrm{H}$ bonds, suggesting stability against decomposition. We also investigated decomposition into diamond-structure $\mathrm{Si}$ and molecular hydrogen at zero pressure, simple hexagonal silicon [15] and Cmca-structure hydrogen [17] phases up to $40 \mathrm{GPa}$, and face-centered cubic silicon and $\mathrm{Cmca}$-structure hydrogen at higher pres- 
sures. While the $\mathrm{O} 3$ phase of Ref. [4] was found to be unstable with respect to decomposition into face-centered cubic silicon and $\mathrm{Cmca}$ hydrogen at its metallization pressure, our $I 4_{1} / a$ and $C 2 / c$ structures are stable with respect to such a decomposition.

We now discuss the possibility of phonon-mediated superconductivity in the $C 2 / c$ phase. Within the BCS theory, the superconducting transition temperature is determined by the density of states at the Fermi energy of the normal state, the Debye temperature of the phonons, and the effective electron-phonon coupling parameter. We estimated the densities of states at the Fermi energies of the $C 2 / c$ and $I 4_{1} / a$ phases at their coexistence pressure of 262.5 GPa using DFT methods. As shown in Fig. 2, the $C 2 / c$ phase is a good metal, with a density of states at the Fermi energy of $1.6 \times 10^{-2} \mathrm{eV}^{-1} \AA^{-3}$, which is about $46 \%$ of the free-electron value and is close to the zeropressure value for lead. We decomposed the densities of states into $\mathrm{H}$ - and $\mathrm{Si}$-associated components using a Mulliken population analysis [22]. Figure 2 shows that below the Fermi energy the density of states is a little larger on the $\mathrm{H}$ atoms than on the $\mathrm{Si}$ ones, while above the Fermi energy it becomes progressively larger on the $\mathrm{Si}$ atoms. This is consistent with the fact that the Pauling electronegativity of $\mathrm{H}(2.2)$ is a little larger than that of $\mathrm{Si}$ (1.9). The fact that the density of electronic states at the Fermi energy projected onto the $\mathrm{H}$ atoms is large suggests that the coupling between the electrons close to the Fermi energy and phonon modes involving motions of the $\mathrm{H}$ atoms may be significant. We also performed DFT calculations of the $\Gamma$-point phonon modes of the $C 2 / c$ phase at $262.5 \mathrm{GPa}$, finding the highest phonon frequency to be $2500 \mathrm{~cm}^{-1}$. This corresponds to an estimated Debye temperature of $3600 \mathrm{~K}$, which is about 40 times larger than that of lead at zero pressure. An examination of the phonon eigenvectors indicates that the highest-frequency modes correspond largely to motions of the $\mathrm{H}$ atoms. The very large Debye temperature and the substantial density of states at the Fermi energy suggest that the $C 2 / c$ phase might exhibit high-temperature superconductivity, but one should be cautious about this conclusion. A reliable estimate of the superconducting transition temperature within the BCS theory requires evaluation of the full electronphonon coupling matrix elements, which is beyond the scope of this Letter.

In summary, we have used a random searching strategy in conjunction with first-principles electronic structure computations to predict the stable high-pressure phases of silane. Our calculations indicate that a first-order phase transition occurs from an insulating/semimetallic phase to a good metal at a pressure achievable within a diamondanvil cell and that the metallic phase might exhibit hightemperature superconductivity.

C. J.P. was supported by the EPSRC. We thank Peter Littlewood for useful discussions and Keith Refson for assistance in performing the phonon calculations.

[1] E. Wigner and H. B. Huntingdon, J. Chem. Phys. 3, 764 (1935).

[2] C. Narayana et al., Nature (London) 393, 46 (1998).

[3] N. W. Ashcroft, Phys. Rev. Lett. 92, 187002 (2004).

[4] J. Feng et al., Phys. Rev. Lett. 96, 017006 (2006).

[5] All of the densities of electronic states referred to in this Letter are per unit volume.

[6] J. Bardeen, L. N. Cooper, and J. R. Schrieffer, Phys. Rev. 108, 1175 (1957).

[7] R. Martonak, A. Laio, and M. Parrinello, Phys. Rev. Lett. 90, 075503 (2003).

[8] S. Yoo and X. C. Zeng, Angew. Chem., Int. Ed. Engl. 44, 1491 (2005).

[9] S. Goedecker, W. Hellmann, and T. Lenosky, Phys. Rev. Lett. 95, 055501 (2005).

[10] A. R. Oganov, C. W. Glass, and S. Ono, Earth Planet. Sci. Lett. 241, 95 (2006).

[11] M. D. Segall, P. J. D. Lindan, M. J. Probert, C. J. Pickard, P. J. Hasnip, S. J. Clark, and M. C. Payne, J. Phys. Condens. Matter 14, 2717 (2002).

[12] J. P. Perdew and A. Zunger, Phys. Rev. B 23, 5048 (1981).

[13] D. Vanderbilt, Phys. Rev. B 41, 7892 (1990).

[14] J. P. Perdew, K. Burke, and M. Ernzerhof, Phys. Rev. Lett. 77, 3865 (1996).

[15] A. Mujica, A. Rubio, A. Muñoz, and R. J. Needs, Rev. Mod. Phys. 75, 863 (2003).

[16] M. T. Yin, Phys. Rev. B 30, 1773 (1984).

[17] K. A. Johnson and N. W. Ashcroft, Nature (London) 403, 632 (2000).

[18] D. J. Wales, Energy Landscapes (Cambridge University Press, Cambridge, England, 2003), Chap. 6.

[19] R. J. Needs, J. Phys. Condens. Matter 11, 10437 (1999).

[20] See EPAPS document No. E-PRLTAO-97-039631 for a table detailing the structures discussed in the main manuscript. For more information on EPAPS, see http:// www.aip.org/pubservs/epaps.html.

[21] L. Burnelle and J. J. Kaufmann, J. Chem. Phys. 43, 3540 (1965).

[22] M.D. Segall, R. Shah, C. J. Pickard, and M. C. Payne, Phys. Rev. B 54, 16317 (1996). 